\title{
An Innovative Application of Big Data in Healthcare: Driving Factors, Operation Mechanism and Development Model
}

\author{
Tao DAI ${ }^{\mathrm{a}, \mathrm{b}, 1}$, Qinkun CHEN ${ }^{\mathrm{b}}$, Liqin XIE ${ }^{\mathrm{b}}$ and Hongpu HU ${ }^{\mathrm{b}}$ \\ a Development Center for Medical Science \& Technology, National Health Commission \\ of the People's Republic of China \\ ${ }^{\mathrm{b}}$ Institute of Medical Information, Chinese Academy of Medical Sciences/Peking Union \\ Medical College, China
}

\begin{abstract}
This paper aims to build a conceptual framework for the innovative application in big data in healthcare. Method: Diamond Model and PEST Model were used to analyze the key driving factors of big data in healthcare through external and endogenous driving factors. Based on this, the operation mechanism and possible development model of big data in healthcare were analyzed by using the diffusion theory of policy innovation. Results: The key factors of innovation and application of big data in healthcare are divided into external and endogenous driving factors. The driving mechanism is defined as "learning, compulsion, competition, imitation and inducement". The conceivable development model is based on big data value innovation and the motivation of different stakeholders. Conclusion: Through the logical analysis of value innovation, the internal relationship among the relevant factors, driving forces and interaction of big data in healthcare application is established, and finally a theoretical analysis framework for innovative application of big data in healthcare is presented.
\end{abstract}

Keywords. Big data in healthcare, innovation application, driving factors, operation mechanism, development model

\section{Introduction}

The development and application of big data in healthcare will enable to increase the supply of health and medical resources, reduce medical costs, improve the quality and efficiency of medical services, and have a important impact on social and economic development and public living standards[1]. At present, big data in healthcare has been gradually applied in clinical medicine, public health, medical insurance and other fields, providing important support for decision-making to medical staff, patients and insured[2]. The purpose of this paper is to build a theoretical framework for innovative application of big data in healthcare. With data value as the main line, the external and endogenous drivers of big data in healthcare application are analyzed from the front end. Whereas, the operation mechanism of big data in healthcare application as the core, the back end focuses on the development mode of application, forming a healthy

${ }^{1}$ Corresponding Author, 2 and 5/F, block B3, building 5, No. 9 Chegongzhuang Street, Xicheng District, Beijing, China; Email: dai.tao@imicams.ac.cn 
complete chain of big data in healthcare application analysis. This innovative application of big data in healthcare provides essential theoretical support[3].

\section{Method}

This study mainly applies Diamond model and PEST model to analyze the key drivers of big data in healthcare through external and endogenous drivers. Based on this, it uses the theory of policy innovation diffusion to analyze its operation mechanism and development mode.

\subsection{Diamond model}

The analysis factors of diamond model include micro forces like production, demand, industry strategy and supporting industry[4]. By comparing the above four aspects of big data in healthcare application, this study analyzes and summarizes the main influencing factors of two major bodies (public sector and market forces) of health big data.

\subsection{PEST analysis}

PEST analysis refers to the macro factors affecting the development of an industry from four aspects of politics, economy, technology and society. By using PEST to analyze the big data in healthcare, we can better grasp the macro environment and the future development of big data in healthcare.

\subsection{Diffusion theory of policy innovation}

The diffusion theory of policy innovation expounds the source of public policy by analyzing the influence of external factors on public policy making. This study examines the operation mechanism of big data in healthcare application by analyzing the learning, competition, imitation and enforcement mechanisms in the process of big data in healthcare application innovation[5][6].

\section{Results and Analysis}

\subsection{Key drivers of big data in healthcare innovation application}

According to the Diamond model and PEST analysis, the key driving factors of big data in healthcare application are divided into external and endogenous driving factors[7]. The external factors mainly include government, economic, social, technical and market factors. The endogenous drivers include development motivation and development capacity and are illustrated in Figure 1. 


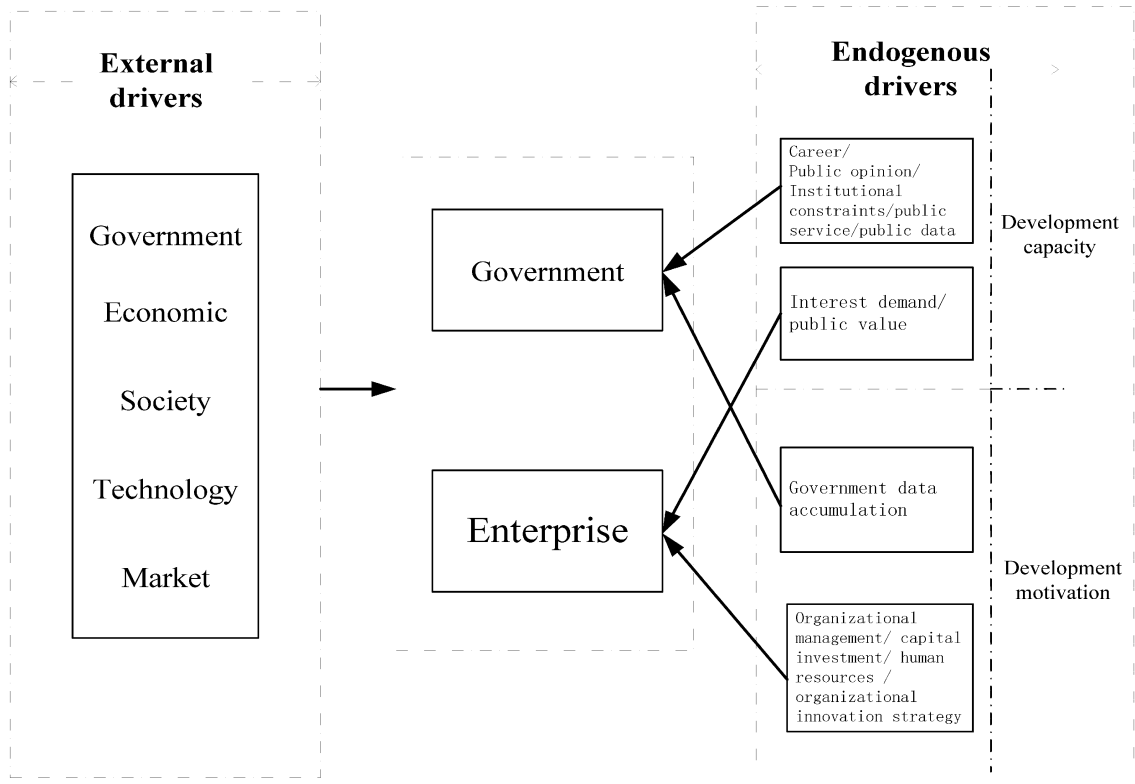

Figure 1.Key drivers for application of big data in healthcare.

- External drivers

Government factor means that the government regulates and guides the resources, technologies and applications of big data in healthcare through various legislations and regulations, policies, standards and other tools[8]. Economic factors are the macroeconomic conditions of a country or a region, such as economic development level, economic structure, residents' income, consumption structure, etc. The impact of social factors on big data in healthcare is more reflected in literacy, employment, community safety, and moral constraints. Technology factor is the main factor and important support for the application and development of big data in healthcare. Technology innovation can promote the core competitiveness of the enterprises, and provide a realistic basis for the development and growth of big data in healthcare industry[9]. On the other hand, market factors like the number of consumers, market size, market structure and market demand, which affect the application direction and industrial scale of big data in healthcare to a certain extent[10].

- Endogenous drivers

The endogenous drivers refer to various influencing factors and conditions arising within the in fields of big data in healthcare and closely affect its operation activities. The logic behind the development motivation, capacity of the public sector and market forces is as follows[11].

The internal and external stakeholders and environmental factors of the public sector will jointly shape the motivation and ability of the public sector to apply health big data. From the perspective of motivation, what are the main leaders' and department leaders' understanding of big data application? It is the key factor that affects the application of big data in healthcare in the public sector. The main factors include career promotion, public opinion promotion, institutional constraints, public 
service motivation and public data promotion[12]. From the characteristics of big data in healthcare, the development capacity of the public sector mainly includes the accumulation of government data.

The motive of market power development is interest demand and public value. The main body of market power's development ability in the application of big data in health care includes four aspects: organization and management mode, capital investment scale, human resources and innovation strategy of interest body[13]. Stakeholder organization and management mode refers to the responsibility structure and management mode based on the specific rules and procedures in order to effectively allocate various resources within the stakeholders (i.e. public sector and market forces). Capital investment scale especially, for big data in healthcare has the high-tech characteristics of emerging industries, which means that it needs a certain scale of capital-intensive R \& D activities to support. Human capital is the most predominant factor, since the big data in healthcare industry is a high-tech knowledge intensive industry and its development is based on the accumulation and assimilation of technology and knowledge[14]. In addition, the stakeholder innovation strategy is to improved based on the environmental changes in order to leverage their own advantages in the competitions.

\subsection{Operation mechanism of big data in healthcare innovation application}

The aforesaid analysis of the key drivers, the external drivers and endogenous factors of different stakeholders have been clarified. However, the mechanism by which these drivers have an effect on the innovative application of big data in healthcare is not yet clear. Based on the four mechanisms of "learning, competition, imitation, and compulsory" in the theory of policy innovation diffusion[15], the driving mechanism for the innovative application of big data in healthcare is defined in the following five mechanisms (Figure 2).

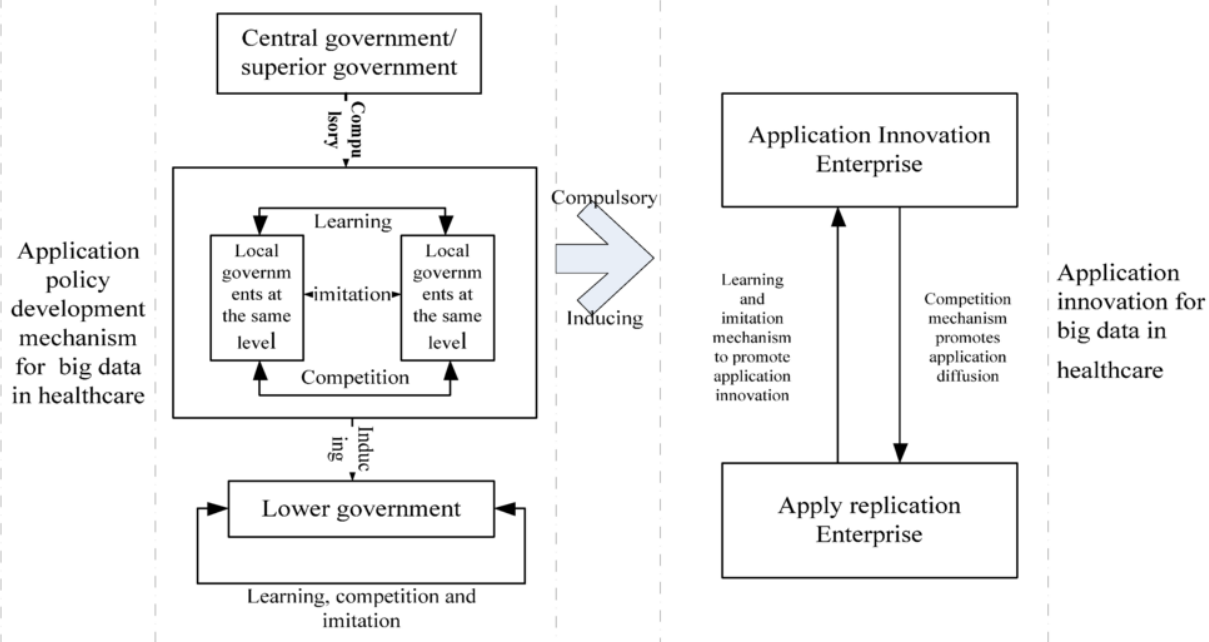

Figure 2. Operation mechanism of application development for big data in healtheare. 
- $\quad$ Learning mechanism

Learning mechanism is a process of information obtaining, interpretation and usage, which explores new solutions to problems through keeping trying[16]. From the government's point of view, learning from the experience of other places can change the thinking mode of decision-makers, so as to provide new and feasible ways or programs to solve their own problems. From the perspective of the enterprises, the importance of learning mechanism is to provide information support for enterprise decision-making, so as to achieve the goal of maximizing their own interests[17]. In the process of innovative application of big data in health care, innovation pioneers can promote the establishment of learning mechanism, and the benefits obtained through learning and innovation can reduce the concerns of relatively conservative people, thus reducing the resistance to reform.

- Compulsory mechanism

At the government level, compulsory mechanism refers to a process in which one actor try to impose its approved policy scheme on another[18]. For example, it directly promotes the innovative implementation of big data in healthcare through administrative orders, so as to improve the possibility of its promotion and application. Therefore, like other policy innovation diffusion, innovation of big data in healthcare often follow the top-down policy implementation path. Power inequality is an important feature of compulsory mechanism[19]. The actors with greater power will affect the decision-making of the relatively weak one. At the enterprise level, the compulsory mechanism comes from market competition. Enterprises must develop new products to meet market demand, or improve management and technical level to reduce costs, so as to improve their own efficiency and competitiveness. And the innovation of these products, management and technologies will promote other backward enterprises to enter the process of compulsory innovation. The compulsory mechanism also further enhances the interaction between the government and the enterprises. The government can push the enterprises to carry out the application and development of the corresponding big data in healthcare through the government documents and other laws. However, the enterprises actions will not fully reflect the original intention of the government policies, but only choose their own interests, leading deviation of government policy implementation or inefficiency of market[20]. The interaction between government and enterprises can also be achieved through inducement mechanism. The details are discussed below.

- Competition mechanism

From the perspective of the government, the competition mechanism of big data in healthcare application refers to the adaptive pressure between different governments in the competition of political promotion resources and economic scale growth. Therefore, it is necessary to compete for more powerful resources through policy adjustment in the new field of big data in healthcare[21]. Under this mechanism, all regions need to maximize their own interests to carry out policy competition, such as talent introduction, financial revenue and so on. If one region takes the lead in introducing new policies in the application of big data in healthcare, it will get more resources in the development. In order to reduce its own loss in the competition, another region will take similar strategic actions, such as adopting the former's new policies or introducing policies with more potential development. From the perspective of enterprises, the driving force of innovation diffusion in health care big data application comes from the pressure of innovative application, that is, non innovative application enterprises 
choose innovative application to avoid being eliminated in market competition; application innovation diffusion is also driven by traction force, that is, innovative enterprises choose initiative promotion and application in order to maximize the profit of innovative application. To sum up, both the government and the enterprise will adopt different measures to promote or limit the diffusion of health and medical big data application innovation according to the principle of maximizing interests[22].

- Imitation mechanism

The imitation mechanism involved in the classical theory of the diffusion of policy innovation is more about the actors' copying and applying or simply modifying to increase the legitimacy of policy choices and the availability of benefits. On the one hand, for the government, most regions simply apply the new policies without combining their actual conditions and giving full play to their advantages. For example, while developing big data in healthcare, most regions will promote the construction of big data industrial parks, without fully considering their own resource endowment. From the enterprises point of view, enterprises can quickly get a share of the market by simply imitating innovative applications, which may have considerable profits in the early stage of operation[23]. However, the threshold of such simple imitation innovation is low, and the subsequent market competition will lead to short profit cycle, and the final price competition will also make the profit return to normal level, which can not fundamentally changing the profit model of enterprises.

- Inducing mechanism

From the government perspective, inducing mechanism refers to that the innovative application projects of big data in healthcare of lower level government or neighboring government have achieved remarkable results, thus guiding and promoting the higher level government or the neighboring government to make innovative decisions. For the enterprises, the government can also provide support in the application and innovation of health care big data through various forms such as incentives, subsidy, and other financial support. By identifying conducive development conditions created by the government, enterprises can judge whether they are in line with the development strategy of the enterprise or the impact on the future profits of the enterprise, and choose their own policy, so as to promote the development of big data in healthcare application[24].

\subsection{The development mode: innovation application of big data in healthcare}

Based on the analysis of driving factors and operation mechanism, this paper analyzes the application of big data in health care with the core logic of value discovery, value creation and value realization, and summarizes the possible development mode of big data in health care[25].

- Possible development model of value innovation based on big data in healthcare

In public management activities and market economy activities, the development model of big data in healthcare includes value discovery, value creation and value realization[26]. Value discovery refers to discovering the value information of market demand through the changes of political, economic and social factors. Value creation refers to the change of development mode caused by the change of internal environment, which mainly refers to the change of organizational structure, cost, 
process, workflow, working mode, working partnership and other factors. Value realization refers to the change of target customers and the innovation of means, which mainly includes changes in customer relationship, distribution channel and revenue model. According to the above analysis, the possible development modes of big data application in health care can be divided into value proposition innovation mode, key business and process innovation mode, value network reconstruction mode, etc[27].

Value proposition innovation model. "Big data" can provide accurate value proposition to government departments, medical institutions or enterprises due to its infinite potential close to everyone's health level or medical treatment mode, that is, clinical application innovation based on health and medical big data. It has the following characteristics: first, it has an insight into the real needs of human health. Over a long time, medical institutions use limited data or experience for diagnosis and treatment activities[28]. However, the real health needs of individuals are difficult to identify due to their complexity, diversity and variability, which require the empowerment of big data. The second is the real-time, accurate and dynamic positioning of diagnosis and treatment. The real-time personalization of big data in health care can quickly collect, correlate and analyze the data with scattered sources and various formats, which helps medical institutions dynamically capture the real health needs of individuals and accurately delineate the health level of the population. Through big data empowerment, the human health status is accurately subdivided and different diagnosis and treatment schemes are implemented. For example, through the analysis of personal genetic data and molecular data, it is helpful to carry out personalized medicine treatment and boost the reform and development of the medical industry[29].

Value creation and transmission innovation mode. Big data in healthcare is not only the basic tool for application, but also the basic condition for innovation. The innovation mode of value creation and transmission is based on big data in healthcare, which is the key business and process of health care activities innovation and empowerment, namely the "big data" process of health and medical activities[30]. According to the scope of its transformation and influence, it can be divided into: reconstructing medical data information flow through health care big data technology, realizing business process innovation and reengineering; taking health and medical data flow as the core, realizing the deep integration and reform of the business model of medical institutions; increasing the new driving force of big data in health and medical application innovation, and realizing new value exploration And path change, from the big data thinking mode, optimize the existing process, and put forward new methods and ideas to solve the problem[31]. To sum up, the key business and process innovation mode is guided by the actual needs of medical services, integrating the application of big data in health care, focusing on solving the key tasks of public hospital reform, family contract service system, hierarchical diagnosis and treatment, the key and difficult problems in the field of health and mainly through optimizing the health management service process, and promoting the refinement in supervision and decision-making.

Value network reconstruction mode. The innovative application big data in healthcare has greatly reduces the cost and risk of the government, medical institutions, relevant enterprises and the public to own and use other external resources, sharing resources and overflow resources, and provides a technical support for the development and the dissemination of new value[32][33]. The process of value network reconstruction is a process of interaction between multiple participants and multiple 
value chains, which is oriented by the value creation of leading products or original target customers. Stakeholders can also integrate into other value chains and other stakeholders through the characteristics of value communication[34]. The innovative application mode of big data in healthcare can be one or more innovative modes[35]. For example, local governments explore the innovation of medical service mode through establishment of internet hospitals and smart hospitals.

- The possible development model based on the motivation of different stakeholders

The development mode of big data application in healthcare is mainly composed of external and endogenous drivers. Among them, the relationship between the government, society and market among the external drivers is the dominant factor that determines the industrial development model of big data in healthcare. The differences in the power and resources possessed by the government, society, and market within the industry determine the different development models, which in turn lead to the different sources of power, influencing factors, and combinations of innovative applications in the industry. According to the main source of innovation power, the development model of innovative application of big data in healthcare can be divided into four types: government-led, industry-driven, socially-initiated, and mixed development[36].

\section{Conclusion}

This paper analyzes the key driving factors, operation mechanism and possible development mode of health care big data based on the core logic of value discovery, value creation and value realization. On the basis of multiple theories, aiming at the two interest subjects of government departments and enterprises, the paper organically integrates the key driving factors and operation mechanism, and combs out the possible development mode of application through the analysis of value innovation, and establishes the internal relationship among the application related factors, driving forces and interactions of health care big data, finally forming an internal logical connection as depicted in Figure 3.
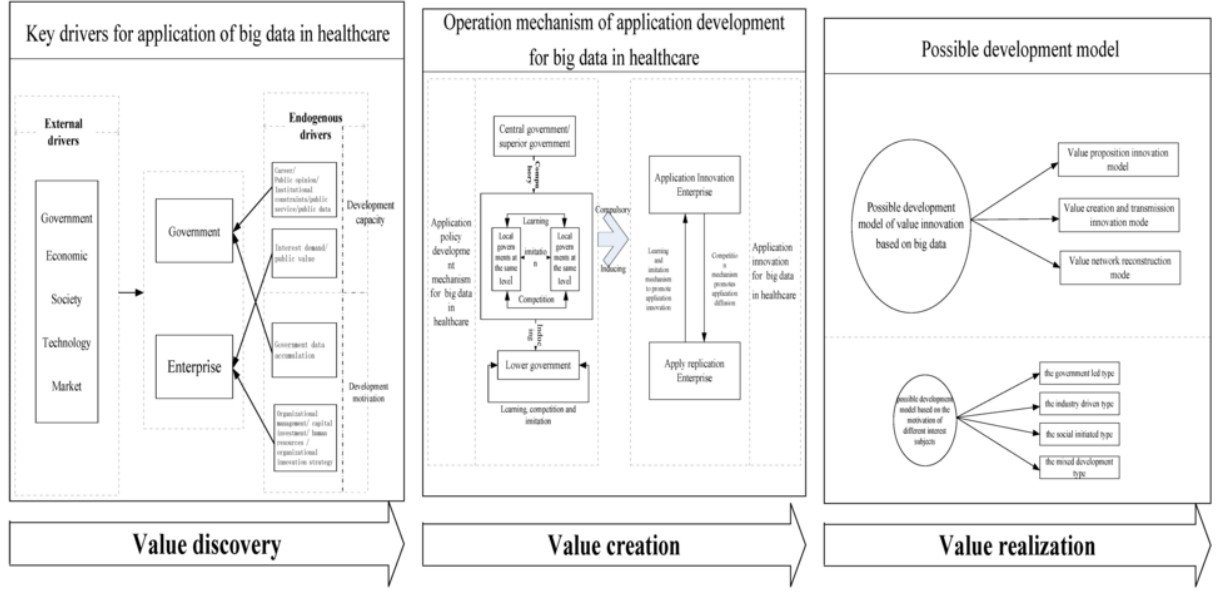

Figure 3. Theoretical analysis frame of big data innovation application in health care. 


\section{References}

[1] Meng Qun, Bi Dan, Zhang Yiming, Yin Xin. Research on the development status and application mode of health care big data. Chinese Journal of health information management, 2016,Jun;13 (06): 547-552.

[2] Yu Qi, Jing Shengjie, Tai Yangfang, et al. Multidimensional analysis of Chinese health care big data policy literature. Chinese Journal of general practice, 2019 Sep ;(09).

[3] Dai Tao. Thinking on the development and application of health care big data . Journal of medical informatics, 2016 Feb;37 (02): 2-8.

[4] Zhou Ying, Liu Yue. Research on Influencing Factors of big data industry development. Modern information, 2017 Aug;37 (08): 129-134.

[5] Yang Daifu. China's policy innovation and diffusion: a basic analysis framework. Local governance research, 2016 Feb; (02): 3-11.

[6] Ma Haiyun, Yang Jinghong. Public governance change driven by big data: basic logic and action framework . China Administration, 2018 Nov;(12): 42-46.

[7] Information Technology - Information Management; Studies from Cape Peninsula University of Technology Add New Findings in the Area of Information Management (A framework for selecting analytics tools to improve healthcare big data usefulness in developing countries). Information Technology Newsweekly,2020,:.

[8] Tiko Iyamu. A framework for selecting analytics tools to improve healthcare big data usefulness in developing countries. SA Journal of Information Management,2020 Jan;22(1):

[9] Meng Tianguang, Zhao Juan. Big data driven intelligent social governance: theoretical construction and governance system . E-government, 2018 Aug; (08): 2-11.

[10] Ma Liang. Motivation, ability and performance of public sector big data application: Theoretical Review and research outlook. E-government, 2016 (4): 62-74.

[11] Fatemeh Soleimani-Roozbahani,Ali Rajabzadeh Ghatari,Reza Radfar. Knowledge discovery from a more than a decade studies on healthcare Big Data systems: a scientometrics study. Journal of Big Data,2019 Jan;6(1):.

[12] Sahoo Prasan Kumar,Mohapatra Suvendu Kumar,Wu Shih Lin. SLA based healthcare big data analysis and computing in cloud network. Journal of Parallel and Distributed Computing,2017,119:.

[13] Wang Lidong,Alexander Cheryl Ann. Big data analytics in medical engineering and healthcare: methods, advances and challenges.. Journal of medical engineering \& technology, 2020.

[14] Famutimi R.F.,Ibitoye A.O.,Ikono R.N,Famutimi T.I.. Comparative Study of Disk Resident and Column Oriented Memory Resident Technique for Healthcare Big Data Management Using Retrieval Time. International Journal of Computer (IJC),2018 Jan;31(1):.

[15] Charles R. Shipan, Craig Volden. The Mechanisms of Policy Diffusion. Blackwell Publishing, 2008,52(4). Charles R. Shipan, Craig Volden. The Mechanisms of Policy Diffusion . Blackwell Publishing, 2008 Mar;52(4).

[16] Ding Mingjie, Zhang Liang. Logical analysis of the formation mechanism of local government policy innovation diffusion. Socialism research, 2014 Mar;(03): 75-82.

[17] Sanghun Lee. The Obesity Paradox in Colorectal Cancer Surgery: An Analysis of Korean Healthcare Big Data, 2012-2013. Nutrition and Cancer,2017 Feb;69(2):.

[18] Deng Ke. Diffusion analysis of big data development policy of provincial government based on the perspective of policy diffusion . Decision consultation, 2019 Mar;(03): 51-58.

[19] Li Wenzhao. Decision making approach of policy process: theoretical basis, evolution process and future prospect. Journal of Gansu University of administration, 2017 Jun; (06): 46-67 + 126-127.

[20] Li Jinyong, Hu Weiqing. Study on the dynamic mechanism of technological innovation diffusion. Research on technology economy and management, 2015 Oct; (10): 19-22.

[21] Md. Ileas Pramanik,Raymond Y.K. Lau,Md. Abul Kalam Azad,Md. Sakir Hossain,Md. Kamal Hossain Chowdhury,B.K. Karmaker. Healthcare informatics and analytics in big data. Expert Systems With Applications,2020,152.

[22] Pastorino Roberta,De Vito Corrado,Migliara Giuseppe,Glocker Katrin,Binenbaum Ilona,Ricciardi Walter,Boccia Stefania. Benefits and challenges of Big Data in healthcare: an overview of the European initiatives..European journal of public health,2019,29.

[23] Joseph Finkelstein MD, PhD,Frederick Zhang BA,Seth A. Levitin BS,David Cappelli DMD, MPH, $\mathrm{PhD}$. Using big data to promote precision oral health in the context of a learning healthcare system. Journal of Public Health Dentistry,2020,80.

[24] Zhu Xufeng, Zhang Youlang. What are the difficulties in the promotion of innovation experience of local governments? A review of the research on the theory of innovation diffusion of public policy . People's forum, academic frontier, 2014 Nova; (17): 63-77.

[25] Zhao Liang. Abnormal drug use detection in electronic health records based on representation learning. East China University of science and technology.2019. 
[26] Li Wenlian, Xia Jianming. Business model innovation based on "big data" . China industrial economy, 2013 May; (05): 83-95.

[27] Deep learning model of chronic disease prediction using healthcare big data. Proceedings of The International Workshop on Future Technology,2019,:

[28] Zoie S.Y. Wong,Jiaqi Zhou,Qingpeng Zhang. Artificial Intelligence for infectious disease Big Data Analytics. Infection, Disease \& Health,2019 Jan;24(1).

[29] Liu Dan, Cao Jiantong, Wang Lu. Research on business model innovation based on big data -- Taking State Grid of China as an example. Contemporary economic management, 2014 Jun;36 (06): 20-26.

[30] Terry, Ken. Is Healthcare Big Data Ready For Prime Time?. Informationweek - Online,2013,:

[31] Li Xiangge, Wang Qiqi, Guo Yibo. Data mining and analysis based on big data era. Electronic production, 2015 Mar; (3): 81 .

[32] Li Yanlin. Research on value chain led business model innovation. Qingdao University of science and technology, 2017.

[33] Pilar Leon-Sanz. Key Points for an Ethical Evaluation of Healthcare Big Data. Processes,2019 Aug;7(8):

[34] Ou Xiaohua. Research on business model innovation of mobile Internet enterprises based on value network reconstruction. Northwest University, 2015.

[35] Wang Qin. Enterprise business model innovation based on value network reconstruction . China industrial economy, 2011 Jan;(01): 79-88.

[36] Liu Yanfei. Research on the development model of health management service industry. Shanghai Academy of Social Sciences, 2016. 